Silva e Almeida. Qualidade na Produção de Refeições...

\title{
QUALIDADE NA PRODUÇÃO DE REFEIÇÕES DE UMA UNIDADE DE ALIMENTAÇÃO E NUTRIÇÃO (UAN).
}

\author{
Caroline de Barros Gonçalves da Silva ${ }^{1}$ \\ Flávia Queiroga Aranha de Almeida²
}

\section{Resumo}

O estudo teve como objetivo avaliar e atuar em uma Unidade de Alimentação e Nutrição (UAN) para aumentar a qualidade e segurança dos alimentos produzidos, a fim de buscar a qualificação da Análise de Perigo e Ponto Crítico de Controle (APPCC), utilizando as Boas Práticas de Fabricação (BPF) como ponto de partida para implementação. Caracteriza-se como um estudo de intervenção, enfatizando a qualidade dos alimentos e a utilização de BPF de uma UAN, localizada em Botucatu, SP. Foi dividido em dois momentos, o primeiro com aplicação de check-list para averiguação das não-conformidades, e o segundo momento um treinamento "in loco" para os colaboradores. A falta de frequiência de higienização das mãos foi vista como um ponto importante e verifica-se que as maiores dificuldades e irregularidades são em relação aos manipuladores que não cumprem as regras. Conseguiu-se aumentar a qualidade e a segurança dos alimentos na unidade, mas estes devem ser mantidos constantemente, para enfim adequar-se ao APPCC.

Palavras chave: Boas práticas de fabricação, nutrição, higiene de alimentos, unidade de alimentação e nutrição.

\section{Introdução}

O risco da ocorrência de doenças de origem alimentar provoca incertezas e preocupações às pessoas que realizam, as refeições fora de casa e exigem um alimento de qualidade; portanto, atualmente e crescente a preocupação do consumidor brasileiro com relação a qualidade dos alimentos e a conseqüente redução de riscos à sua saúde e do meio ambiente (DAMASCENO, 1997). A relação alimento-saúde é portanto, de grande relevância, mostrando a necessidade de desenvolver programas que visem difundir conhecimentos que protejam a população através da orientação aos profissionais do ramo de alimentos quanto aos riscos inerentes ao consumo alimentar. Além de difundir a legislação, esses programas devem sensibilizar o profissional para a adoção de Boas Práticas operacionais na manipulação,

\footnotetext{
${ }^{1}$ Graduanda do Curso de Nutrição do Instituto de Biociências - Universidade Estadual Paulista "Júlio de Mesquita Filho" - UNESP - Campus de Botucatu.

${ }^{2}$ Professora Doutora do Curso de Nutrição do Instituto de Biociências - Universidade Estadual Paulista "Júlio de Mesquita Filho" - UNESP - Campus de Botucatu.
} 
preparo e comércio de alimentos, visando a produção de alimentos mais seguros. Os procedimentos preconizados para garantir um alimento de boa qualidade higiênico-sanitária difundidos através de programas de educação são aplicáveis tanto no preparo profissional quanto no domiciliar, contribuindo para a promoção da saúde e para a melhoria da qualidade de vida do indivíduo e da coletividade (SOUZA et. al, 2003).

FERREIRA et al. (2001) enfatizam a necessidade de constante aperfeiçoamento das ações de controle sanitário na área de alimentos. De acordo com a relevância do controle da qualidade e segurança alimentar o Ministério da Saúde, dentro da sua competência, elaborou as portarias 1428 de 26/12/1993 e 326 de 30/7/1997, que estabelecem as orientações necessárias para inspeção sanitária por meio da verificação do Sistema de Análise de Perigo e Ponto Crítico de Controle (APPCC) da empresa produtora e de serviços de alimentos e os aspectos que devem ser levados em conta para a aplicação de boas práticas de fabricação $(\mathrm{BPF})$, respectivamente.

O Codex Alimentarius (2001) estabelece as condições necessárias para a higiene e produção de alimentos seguros. Seus princípios são pré-requisitos para a implantação do APPCC, em que ocorre o controle de cada etapa de processamento. GALHARDI (2002) confirma as BPF como pré-requisitos fundamentais para a implantação do sistema de APPCC, considerado parte integrante das medidas de segurança alimentar e ponto referencial para produção de normas reguladoras (legislação) da produção de alimentos.

BPF são normas de procedimentos a fim de atingir um determinado padrão de identidade e qualidade de um produto e/ou serviço na área de alimentos, incluindo-se bebidas, utensílios e materiais em contato com alimentos (BRASIL, 1993).

Akutsu et al. (2005) consideram que as BPF possuem quatro pontos principais a serem analisados: termos relevantes - inclusive pontos críticos de controle e práticas referentes à pessoal; instalações - áreas externas, plantas físicas, ventilação e iluminação adequadas, controle de pragas, uso e armazenamento de produtos químicos, abastecimento de água, encanamento e coleta de lixo; requisitos gerais de equipamentos - construção, facilidade de limpeza e manutenção; e controles de produção.

Uma das ferramentas utilizadas para se atingir as Boas Práticas é a ficha de inspeção ou check-list, que possibilita fazer uma avaliação preliminar das condições higiênicosanitárias de um estabelecimento produtor de alimentos. Esta avaliação inicial permite levantar itens não conformes e, a partir dos dados coletados, prever ações corretivas para 
adequação dos requisitos buscando eliminar e reduzir riscos que possam comprometer os alimentos e a saúde do consumidor (SENAC, 2001; GENTA et.al., 2005).

Com o crescimento do mercado de alimentação, torna-se imprescindível criar um diferencial competitivo nas empresas por meio da melhoria da qualidade dos produtos e serviços oferecidos, para que esse diferencial determine quais permanecerão no mercado (AKUTSU et al., 2005).

A partir disso, o estudo teve como objetivo avaliar e atuar em uma UAN para aumentar a qualidade e segurança dos alimentos produzidos, a fim de buscar a qualificação APPCC.

\section{Metodologia}

O trabalho caracteriza-se como um estudo de intervenção, enfatizando a qualidade dos alimentos e a utilização de BPF de uma Unidade de Alimentação e Nutrição (UAN), localizada em Botucatu, SP.

O serviço de alimentação ocorre por uma empresa especializada, conta com 45 colaboradores em 3 turnos. Servindo aproximadamente 1700 refeições (1100 no almoço e 600 no jantar).

$\mathrm{O}$ estudo foi realizado em 2 meses, aplicado apenas no primeiro turno (7h às 15h50) e dividido em dois momentos, o primeiro com aplicação de check-list para averiguação das nãoconformidades e acompanhamento do andamento da unidade, e o segundo momento um treinamento "in loco" para os colaboradores.

O check-list foi formulado com base na Portaria CVS 6/99, Portaria 216 de 15/09/2004 e Manual ABERC (2009). Os principais itens e áreas abordados foram:

- Recebimento e armazenamento de produtos (Estoque): recepção, higiene pessoal, higiene do ambiente, manutenção civil e eletromecânica, armazenamento de produtos não perecíveis, armazenamento de produtos de limpeza e descartáveis, câmaras frias, amostras de referência e registros de recebimento.

- Açougue, área de saladas, confeitaria, cocção: higiene do ambiente, de equipamentos e utensílios, higiene pessoal e das mãos, câmaras frias, processamento de alimentos, manipulação, procedimentos, controle de temperatura, armazenamento, manutenção civil e eletromecânica. 
- Apoio: higiene do ambiente, de equipamentos e utensílios, higiene pessoal e das mãos, armazenamento de utensílios limpos, área de higienização, área de armazenamento de caixas, área de resíduos, área externa, devolução, vestiários, manutenção civil.

O check list, totalizou 130 itens, cada um era verificado se estava de acordo com as normas, se aplicável (S), recebia a nota máxima de conformidade, se não aplicável $(\mathrm{N})$, nota zero. Após a avaliação, somava-se as notas dos itens por área e dividia pelo número de itens averiguados, para estabelecer a média por áreas.

Foi atribuída uma escala de pontuação de 1 a 4, para a classificação das áreas, que significa 1 (crítico - 0 a 30\%), 2 (não conformidades maiores - 31\% a 60\%), 3 (oportunidade de melhoria $-61 \%$ a $80 \%$ ), 4 (pleno cumprimento dos procedimentos $-81 \%$ a $100 \%$ ).

O treinamento "in loco", com duração de 30 dias, foi aplicado em cada área de serviço da UAN, durante todas as atividades de rotina do colaborador. Cada área foi monitorada por 3 dias consecutivos.

Para o treinamento utilizou-se a CARTILHA DA ANVISA - Cartilha sobre Boas Práticas para serviços de alimentação, Portaria CVS 6/99, Portaria 216 de 15/09/2004 e Manual ABERC (2009). A aplicação do treinamento aconteceu no primeiro momento por meio de conversas didáticas e prática em todas as áreas analisadas. E no segundo e terceiro momento, com observações e orientações quando não seguiam as normas.

\section{Resultados e Discussão}

Os resultados demonstram que foram identificadas irregularidades em todas as áreas analisadas, conforme visto na Figura 1. 


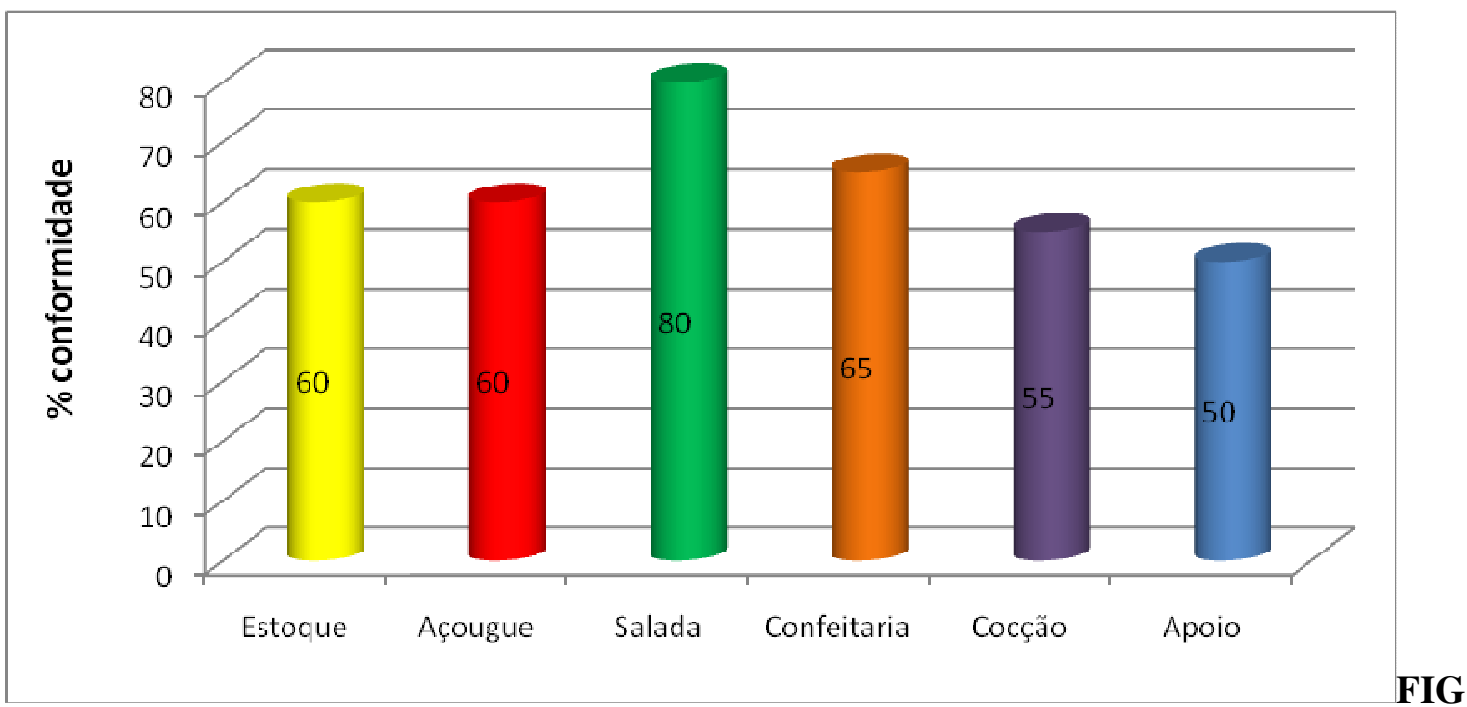

URA 1. Classificação das conformidades encontradas na UAN separada por áreas.

Observa-se que o estoque, o açougue, a cocção e o apoio encontram-se na faixa de não conformidades maiores, e as principais dificuldades encontradas foram em relação à organização, manipulação de alimentos de maneira incorreta, ausência de amostras in natura, controle de temperatura e identificação dos alimentos.

A confeitaria encontrava-se na faixa de oportunidade de melhorias, pois o maior problema foi o cruzamento de alimentos não higienizados (frutas) e já preparados (sobremesas como pudins, gelatinas, bolos), e erros durante a preparação de sobremesas, não seguindo o receituário padrão.

A área da salada foi a que obteve melhores resultados, visto que as dúvidas são em relação a higienização correta de legumes e verduras e a quantidade ideal de cloro para caixas e cubas.

Os resultados encontrados neste trabalho são similares aos resultados encontrados por Akutsu et al. (2005) onde maior atenção deve ser direcionada aos manipuladores de alimentos, os quais são os responsáveis pela produção segura dos mesmos, e a produção de preparações higiênicas e a educação dos manipuladores de alimentos envolvidos na preparação, processamento e serviços são limites cruciais para a prevenção da maioria das doenças veiculadas por alimentos.

Após os resultados encontrados, realizou-se um treinamento "in loco" com os manipuladores para corrigir os problemas encontrados e melhorar as BPF. A figura 2 demonstra as conformidades após o treinamento. 


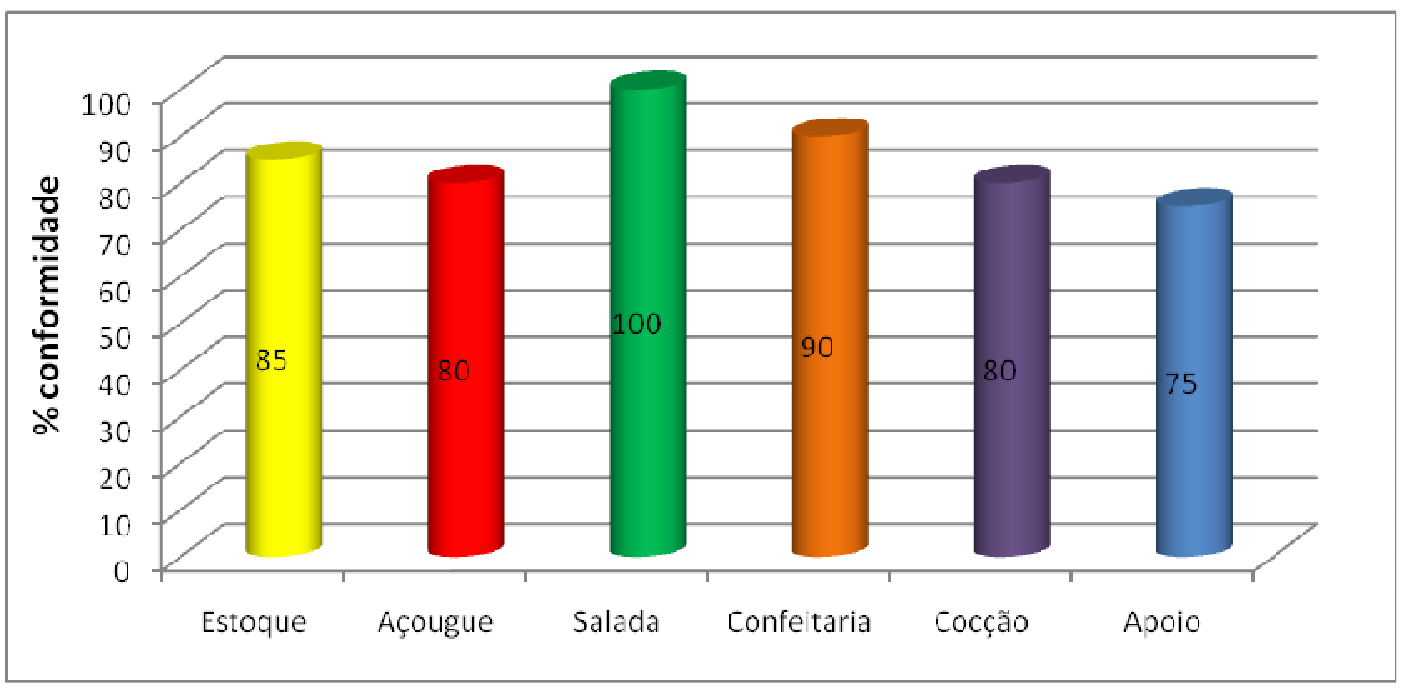

Figura 2. Classificação das conformidades encontradas na UAN após aplicação de treinamento.

Todas as áreas obtiveram melhorias: açougue e salada, de 20\%; estoque, confeitaria, cocção e apoio de $25 \%$.

MORTIMORE (2000) alerta para que a segurança alimentar possa ser implementada, há necessidade de que as pessoas envolvidas, tanto no planejamento de APPCC, quanto na operacionalização, apresentem competência. Portanto, seleção, treinamento e educação dos manipuladores, bem como avaliação de competências, são critérios para o sucesso e alcance do fornecimento de alimento seguro.

Porém treinamento e averiguação de uso de BPF devem ser cobrados diariamente, visto que a rotatividade de colaboradores na unidade é intensa. E a falta de cumprimento das regras, se não forem cobradas, não são realizadas. Por isso propõe-se que a unidade deveria contratar um nutricionista administrador e um nutricionista de produção, para garantir o cumprimento de todos os procedimentos necessários.

\section{Conclusão}

As reflexões que determinam a investigação acerca das condições de produção de alimentos demonstram que a UAN obteve melhores resultados na área de salada e confeitaria, classificando-se em pleno cumprimento dos procedimentos. E o estoque, apoio, açougue e cocção, conseguiram seguir a BPF, mas com pequenas falhas, classificando-se em oportunidade de melhorias. 
Aumentou-se a qualidade e a segurança dos alimentos na unidade, mas estes devem ser mantidos constantemente, para enfim adequar-se ao APPCC, e para isso a conscientização e cumprimento por parte dos colaboradores deve ser cobrada.

\section{Referências}

ABREU, E.S.; SPINELLI, M.G.N.; ZANARDI, A.M.P. Gestão de unidades de alimentação e nutrição: um modo de fazer . $3^{\mathrm{a}}$ ed . São Paulo: Metha, 2009. 342p.

AKUTSU, RC. et al. Adequação das boas práticas de fabricação em serviços de alimentação. Rev. de Nutr., Campinas, v.18, n.3, p. 419-427, 2005.

ASSOCIAÇÃO BRASILEIRA DAS EMPRESAS DE REFEIÇÕES COLETIVAS (ABERC). Manual ABERC de práticas de elaboração e serviço de refeições para coletividades. $9^{\mathrm{a}}$. ed. São Paulo: ABERC, 2009. 221p.

BRASIL. Ministério da Saúde. Agência Nacional de Vigilância Sanitária. Legislação. Resoluções. Resolução no 216, de 15 de setembro de 2004: dispõe sobre o Regulamento Técnico de Boas Práticas para Serviços de Alimentação. Brasília: Diário Oficial da União; 16 setembro 2004. Seção I, p. 24-27.

BRASIL. Portaria n ${ }^{\circ}$ 58/93 de 17 de maio de 1993. Estabelece Diretrizes e Princípios para a inspeção e Fiscalização Sanitária de Alimentos, Diretrizes e Orientações para o Estabelecimento de Padrões de Identidade e Qualidade de Bens e Serviços na Área de Alimentos - Boas Práticas de Produção e Prestação de Serviços; Regulamento Técnico para Estabelecimento de Padrões de Identidade e Qualidade dos Alimentos. Brasília: Diário Oficial da União, 31 maio 1993. Seção I, p. 7228-33.

CARTILHA DA ANVISA - Cartilha sobre Boas Práticas para serviços de alimentação. Acesso em 15/04/2010. Disponível em: http://www.anvisa.gov.br/divulga/public/alimentos/cartilha_gicra.pdf.

CODEX ALIMENTARIUS. Food Hygiene basic texts. 2nd ed. Rome; 2001.

DAMASCENO, K.S.F.S.C. Controle de qualidade de "sanduiches naturais" comercializados em Natal nas lanchonetes da Universidade Federal do Rio Grande do Norte. 1997. 54 f. Monografia (Especialização em Controle de Qualidade de Alimentos, Nutrição e Saúde Publica) - Departamento de Nutrição e Saúde coletiva, Universidade Federal do Rio Grande do Norte, Natal.

FERREIRA, C.E.M; BEZERRA, L.G; NETO, G.V. Guia para implantação de boas práticas de fabricação (BPF) e do Sistema APPCC. Rio de Janeiro; 2001.

GALHARDI, M.G. Boas Práticas de Fabricação. Módulos do centro de excelência em turismo da Universidade de Brasília. Brasília: Universidade de Brasília; 2002. 
GENTA, T.M.S.; MAURICIO, A.A.; MATIOLI, G. Avaliação das Boas Praticas através de check-list aplicado em restaurantes self-service da região central de Maringá, Estado do Paraná. Acta Scientiarum Health Eciences, Maringá, v.27, n.2, p.151-156, 2005.

MORTIMORE, S. An example of some procedures used to assess HACCP systems within the food manufacturing industry. Food Control., v.11, p. 403-13, 2000.

SÃO PAULO (Estado). Portaria ${ }^{\circ}$ CVS - 6, de 3 de março de 1999: aprova o Regulamento Técnico que estabelece os Parâmetros e Critérios para o Controle Higiênico-Sanitário em Estabelecimentos de Alimentos. São Paulo: Diário Oficial do Estado, 12 mar. 1999, Seção I, p.24-7.

SERVICO NACIONAL DE APRENDIZAGEM COMERCIAL - SENAC. Manual de elementos de apoio para o sistema APPCC. Rio de Janeiro: SENAC/Departamento Nacional, 2001.

SOUZA, S. S.; PELICION I, M. C. F.; PEREIRA, I. M. T. B. A vigilância sanitária de alimentos como instrumento de promoção de saúde. Higiene Alimentar, São Paulo, v. 17, n. 113, p. 33-37, out. 2003.

WALKER E., PRITCHARD C., FORSYTHE S. Hazard analysis critical control point and prerequisite programme implementation in small and medium size food businesses. Food Control., v.14, p.169-174, 2003.

\title{
QUALITY IN THE PRODUCTION OF MEALS FOR FOODSERVICE AND NUTRITION UNITS
}

\begin{abstract}
This study aimed to evaluate the food production and then act to increase the quality and security of a service, in order to meet the requirements for qualification HAPPC, using good manufacturing procedures as starting point for implementation. It is characterized as an intervention study, emphasizing the quality of foods and the use of good manufacturing procedures at the foodservice and nutrition units, located in Botucatu-SP. It was composed of 2 moments. In the first, we applied a check-list for ascertainment of non-conformity and in the second moment a training "in loco" was given for the collaborators. The lack of hygienic cleaning of hands is seen as an important negative point. We verified that the biggest difficulties and irregularities are related to the manipulators which don't follow the rules. Good manufacturing procedures were obtained to increase the quality and the security of foods in the unit, but these must be kept constantly adjusted to the standards.
\end{abstract}

Keywords: good manufacture procedures, nutrition, food hygiene, foodservice and nutrition units. 